\title{
A Quadrature Filter Approach for Registration Accuracy Assessment of Fundus Images
}

\author{
Kedir M. Adal ${ }^{1,3}$, Rosalie Couvert, D.W.J. Meijer, Jose P. Martinez² \\ Koenraad A. Vermeer ${ }^{1}$, L.J. van Vliet $^{3}$ \\ 1 Rotterdam Ophthalmic Institute, Rotterdam The Netherlands \\ 2 Rotterdam Eye Hospital, Rotterdam, The Netherlands \\ 3 Quantitative Imaging Group, Department of Imaging Physics, \\ Delft University of Technology, Delft, The Netherlands
}

\begin{abstract}
This paper presents a method to automatically assess the accuracy of image registration. It is applicable to images in which vessels are the main landmarks such as fundus images and angiography. The method simultaneously exploits not only the position, but also the intensity profile across the vasculatures. The accuracy measure is defined as the energy of the odd component of the $1 \mathrm{D}$ vessel profile in the difference image divided by the total energy of the corresponding vessels in the constituting images. Scale and orientation-selective quadrature filter banks have been employed to analyze the 1D signal profiles. Subsequently, the relative energy measure has been calibrated such that the measure translates to a spatial misalignment in pixels. The method was validated on a fundus image dataset from a diabetic retinopathy screening program at the Rotterdam Eye Hospital. An evaluation showed that the proposed measure assesses the registration accuracy with a bias of -0.1 pixels and a precision (standard deviation) of 0.9 pixels. The small Fourier footprint of the orientation selective quadrature filters makes the method robust against noise.
\end{abstract}

Keywords: Registration validation, vessels, eye fundus, diabetic retinopathy.

\section{Introduction}

Registration of medical images can be defined as a spatial mapping between two or more images in order to relate them for diagnosis, screening, or other clinical purposes. The images to be registered may be acquired from different patients and can be of different imaging modalities. However, they can also come from longitudinal data of the same patient to detect pathologies or to quantify disease progression. Depending on the task at hand one chooses a specific registration method. The state-of-the-art medical image registration techniques are presented in recent surveys $[1,2]$. In addition to robustness, a key performance measure of these techniques is accuracy.

In medical images where sparsely distributed blood vessels are the main available landmarks, quantifying the registration accuracy is challenging. The 
most common way to quantify the registration accuracy of such images is to use the vessels' skeleton in the two images and evaluate the distance between them [3]. However, extracting the skeleton is not a trivial task. Some parts may be missing in one of the images and it is also sensitive to noise. Moreover, it does not provide sub-pixel resolution and results in the loss of all the valuable intensity information. Conventional metrics such as intensity difference, cross correlation, and mutual information fail either to handle differences in contrast or defocus between registered image pairs. In addition, these metrics do not provide the spatial registration error in pixels, which is crucial to determine if the registration result meets the required level of accuracy for a certain application.

Other evaluation approaches include visual inspection of the registration result in an overlay mode, comparing the obtained transformation with the "ground truth" transformation, or testing its transitivity [4,5]. Visual inspection is a very fast way to find large registration errors. As such, it is useful for determining the robustness of an algorithm and to find outliers, but it is not suited for a quantitative assessment of the accuracy for images with small registration errors. Since a ground truth is often not available, one has to simulate a given transformation to use this method for evaluation. Simulated transformations, however, will only cover those deformations that are part of the model and will therefore miss some of the deformations encountered in practical cases. An alternative is to evaluate the transitivity of the algorithm, but this does not guarantee a high registration accuracy because the registration errors may be correlated. Another major limitation common to all of the above approaches is that they do not provide an objective error measure in pixels.

In this paper, we address the problem of assessing the registration accuracy of images in which the vasculature is the main feature. The proposed method uses the vessels as landmarks to quantify the accuracy of the alignment in the direction perpendicular to the vessels. The presence of vessel branches with various orientations ensures a complete accuracy assessment. Thus, the proposed mismatch measure is a quantity related to the physical displacements occurring across the vessels.

A scale and orientation adaptive quadrature filter bank has been used to decompose the $1 \mathrm{D}$ profile perpendicular to a vessel in the difference image into an odd and an even component. The ratio of the energy of the odd component to the total energy of the vessel profile of the two images provides a measure that is directly related to the registration error. This measure is invariant to other disturbing factors due to imaging and illumination artifacts.

We applied our method to assess the accuracy of registered red-free fundus photos acquired for diabetic retinopathy screening. We show that the proposed error measure is strongly related to the spatial registration error in the registered images, thus it can be used as a tool in the longitudinal screening of fundus images for disease progression. 


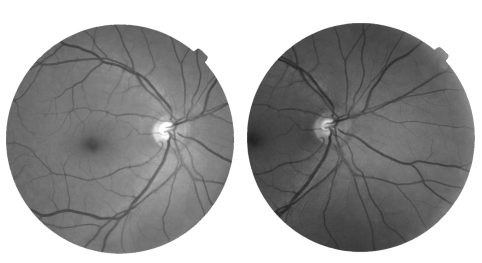

(a)

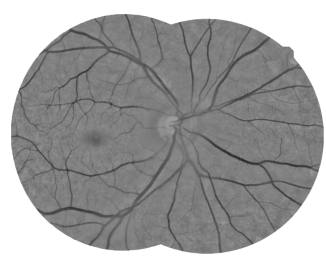

(b)

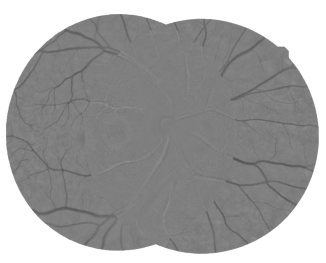

(c)

Fig. 1: Example of registered fundus image pairs. (a) Macula and optic nervecentered images. (b) Normalized and registered output image. (c) The difference image in the overlap region. Zero difference is depicted in grey.

\section{Material and Method}

\section{$2.1 \quad$ Material}

The proposed algorithm was validated on fundus images obtained from an ongoing diabetic retinopathy screening program at the Rotterdam Eye Hospital. 20 diabetes patients who visited the hospital in two consecutive years for diabetic retinopathy screening were included. During each visit, four images of maculacentered, optic nerve-centered, superior, and temporal regions of the retina were acquired from each eye. For the sake of simplicity, we will use macula and optic nerve-centered images of each patient.

\subsection{Registration Method}

Although the proposed quantitative accuracy assessment method can be applied to evaluate the accuracy of any registration method for images with vessels, we demonstrate it here by applying it to a hierarchical non-rigid fundus image registration approach [6]. The method registers image pairs using intensity as well as structural information of the retinal vasculature after normalization of the green channel for luminosity and contrast variation over the full field of view. The normalized images are registered based on a vasculature-weighted mean square difference (MSD) similarity measure and a multiresolution matching strategy coupled with a hierarchical registration model. Figure 1a and 1b show an example of individual image pairs and the registered normalized mosaic. Figure 1c shows the difference image in the overlap region.

\subsection{Registration Accuracy Assessment}

When evaluating the registration accuracy of medical images in which the vasculature is the main feature, it is of importance to be able to differentiate vessel misalignments (Fig.2d) from other contributions to the MSD such as contrast differences (Fig.2b) and (de)focus differences (Fig.2c). 
a)
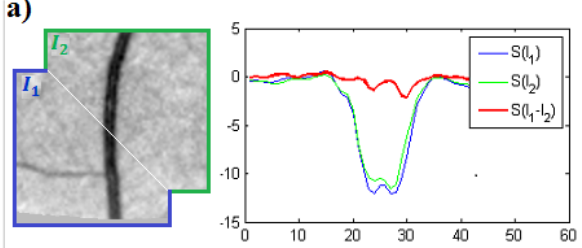

c)

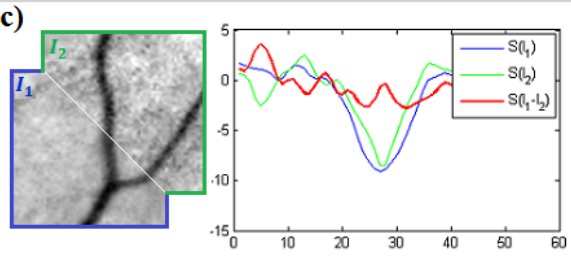

b)

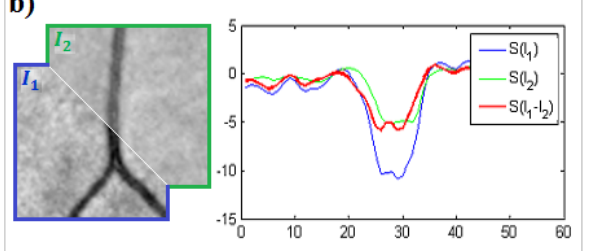

d)

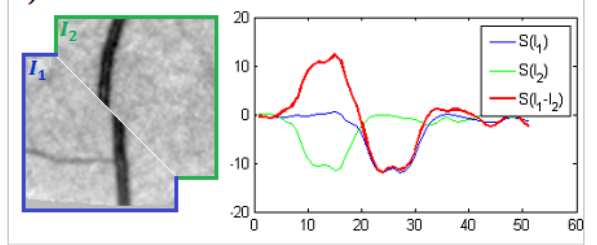

Fig. 2: Examples of registered image patches with the corresponding vessel profiles and the difference profile. (a-c) Correct alignment. (d) Misalignment.

In cases where the alignment is correct (Fig.2a-2c), the profiles of the difference image perpendicular to the aligned vasculatures shows an even signal whereas a misalignment yields an odd (Fig.2d) signal. Hence, a new measure called relative vessel misalignment energy (RVME) which exploits this signal property of the difference image is defined to assess the registration accuracy. The RVME measure is expressed as the energy of the odd component of the vessel profile in the difference image divided by the total energy of the two corresponding vessel profiles:

$$
R V M E=\frac{E\left(S_{o d d, \perp}\left(I_{d i f f, p}\right)\right)}{E\left(S_{\perp}\left(I_{1, p_{1}}\right)\right)+E\left(S_{\perp}\left(I_{2, p_{2}}\right)\right)},
$$

where $E\left(S_{o d d, \perp}\left(I_{d i f f, p}\right)\right)$ is the energy of the odd component of the difference image signal profile perpendicular to the vasculatures and centered at a point $p$. $E\left(S_{\perp}\left(I_{1, p_{1}}\right)\right)$ and $E\left(S_{\perp}\left(I_{2, p_{2}}\right)\right)$ are the energies of the signal profiles perpendicular to the vasculatures of the registered image pairs $I_{1}$ and $I_{2}$ centered at $p_{1}$ and $p_{2}$, respectively.

Quadrature filters. In order to compute the RVME, the odd and even parts of the signal perpendicular to the vessel need to be extracted. A quadrature filter $q(x)$ gives the analytic representation of a signal that has been filtered by a filter $h(x)$. Such a filter is defined as $q(x)=h(x)+i \cdot \mathcal{H}(h(x))$, where $\mathcal{H}$ is the Hilbert transform.

By choosing an even filter $h(x)$, the real and imaginary parts of $q(x)$ allow to differentiate between even and odd profile signals, respectively. Therefore, the analytic representation $f$ of the profile signal $S$, centered at a given point, becomes:

$$
f=S(x) * q(x)=S(x) * \operatorname{Re}(q(x))+i \cdot S(x) * \operatorname{Im}(q(x))
$$


If the profile signal is even, then the response to the filter will be real. However, the response to an odd signal profile will be imaginary, which is in agreement with the models in figure 2 . We can thus use this method to evaluate the RVME.

Orientation space. As the Hilbert transform needs to be applied to the signal in a certain direction, we adopted an orientation space filter bank $[7,8]$. The filter bank is composed of rotated versions of an orientation selective quadrature filter. The orientation selectivity and scale selection can be best described in the frequency domain representation of the filter [8]:

$$
\Phi(\theta, w)=\underbrace{\exp \left(-\frac{(N \theta)^{2}}{2 \pi^{2}}\right)}_{\text {angular filter }} \cdot \underbrace{\left(\frac{|w|}{w_{c}}\right)^{\frac{w_{c}^{2}}{b_{w}^{2}}} \exp \left(-\frac{w^{2}-w_{c}^{2}}{2 b_{w}^{2}}\right)}_{\text {radial filter }},
$$

where $\theta$ is the orientation angle, $w$ the radial frequency, $N$ the number of filters in the bank which defines the angular resolution, $w_{c}$ the frequency at which the filter attains its maximum, and $b_{w}$ the standard deviation of the radial Gaussian. These parameters were set based on the vessels' width and orientation.

The $N$ filtered images are relatively noise-free and the signal in each image represents a specific orientation range, solving the problem of spurious or missing vessel skeleton and simplifying the computation of the RVME. Eq.(1) becomes:

$$
R V M E=\frac{E\left(\operatorname{Im}\left(I_{\text {diff }} * q_{\widehat{\theta}}\right)\right)}{E\left(I_{1} * q_{\widehat{\theta}}\right)+E\left(I_{2} * q_{\widehat{\theta}}\right)}
$$

where $q_{\widehat{\theta}}$ is the spatial domain representation of the orientation space filter corresponding to the orientation of the vessel.

Vessel detection and width estimation. In order to match the scale and orientation of the quadrature filters, each vasculature along with its width and orientation must be identified. The vasculature region is first detected from one of the registered images using a multi-scale $(\sigma \in[1,9]$ pixels) vessel enhancement approach [9] followed by connected component analysis . A vasculature mask is then obtained by keeping objects larger than 2000 pixels, discarding possible noise. This mask is further reduced to a skeleton, and its junctions are removed, leaving only segments of the skeleton along which the registration assessment can be done. It should be noted that even though we used the skeleton, the assessment can also be done on any point along the vessel segment, solving the requirement of accurate skeleton detection.

Once the skeleton is detected, the width and orientation of the vasculature segments are estimated by exploiting the properties of the local principal curvature computed from the second-order derivatives of the image. Given a scalenormalized Hessian matrix of each pixel $\mathbf{x}=(x, y)$ :

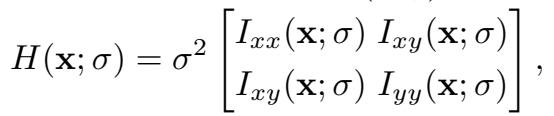

where each element $I . .(\mathbf{x} ; \sigma)$ is the convolution of the image at location $\mathbf{x}$ with a second-order Gaussian derivative kernel of scale $\sigma$ along the specified subscript . 
For each vasculature pixel, the eigenvalues $\lambda_{1}$ and $\lambda_{2}\left(\left|\lambda_{1}\right| \gg\left|\lambda_{2}\right|\right)$ of $H$ correspond to the local (intensity) curvature values across and along a vessel, respectively. The width is approximated by selecting the scale $\hat{\sigma}$ which maximizes the largest principal curvature $\lambda_{1}$ :

$$
\hat{\sigma}=\arg \max _{\sigma} \lambda_{1}(\sigma)
$$

Hence, at each evaluation point, the quadrature filters were tuned to match the vessel width $(2 \hat{\sigma})$ and the orientation of the vasculature, determined by the eigenvector corresponding to $\lambda_{1}(\hat{\sigma})$.

\section{Experiments and Results}

\subsection{Parameter Optimization}

The orientation space filter bank (Eq. 3) parameters $\left(N, w_{c}, b_{w}\right)$ were optimized to match the width and orientation of vessels. The angular resolution $N$ was set to 15 and the optimal values of the remaining parameters are summarized as $w_{c}=b_{w}=0.15 \hat{\sigma}^{-1}$. These parameters were the same for all fundus data, healthy and diseased.

\subsection{Evaluation}

In order to determine the relation between the RVME measure and the spatial registration accuracy, an evaluation was done on registered image pairs. For each pair, a mismatch was introduced by translating one of the image pairs horizontally (but any other direction would suffice as well) by a known amount before calculating the RVME for vessels running perpendicular to the imposed displacement. The translation was increased by 1 pixel until the vessels were fully misaligned. Figure 3a shows the evaluation results stratified by vessel width. Since the orientation selective filters have footprint that runs parallel to vessels, the result of neighboring pixels are correlated, thus evaluation was done at randomly sampled points uniformly distributed across the entire overlap region. Each point on the graph is the average over 50 evaluation points selected from the 20 registered fundus image pairs.

The results show a strong correlation between the RVME measure and the imposed misalignment (registration error). Moreover, figure 3b shows the RVME measure of various vessel widths have an approximately linear correlation, which indicates that it is robust to variation in the vessel width. Hence, given the RVME value and the estimated vessel width, the spatial registration accuracy can be determined in a straightforward manner. This evaluation showed that the proposed measure assesses the registration accuracy with a bias of -0.1 pixels and a precision (standard deviation) of 0.9 pixels.

In the example shown in figure 4 , even if the registration of two images is perfect, the difference image at the location of blood vessels may show a significant residual signal. For example, in figure 4a noise leads to an MSD of 0.78. In figure $4 \mathrm{~b}$, the contrast difference between correctly aligned vessels leads 


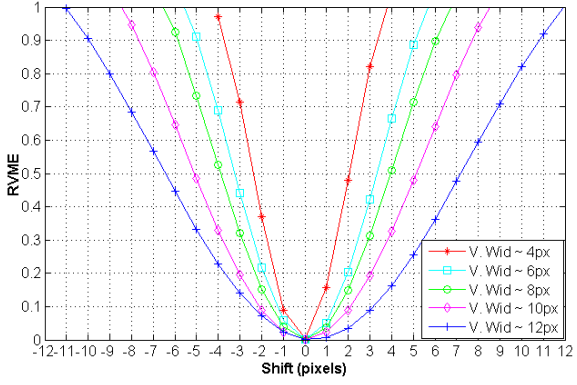

(a)

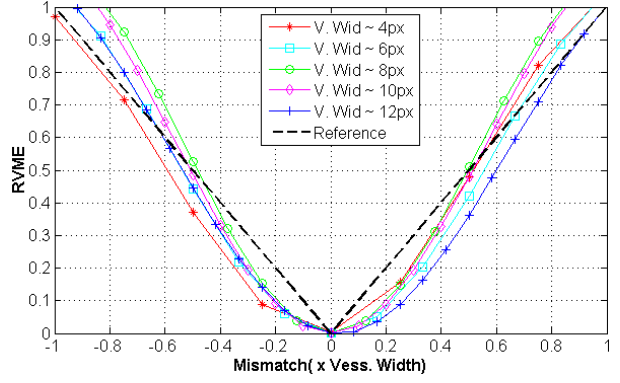

(b)

Fig. 3: Registration assessment results. (a) RVME as a function of misalignment in pixels. (b) Correlation between RVME and misalignment as a function of vessel width.
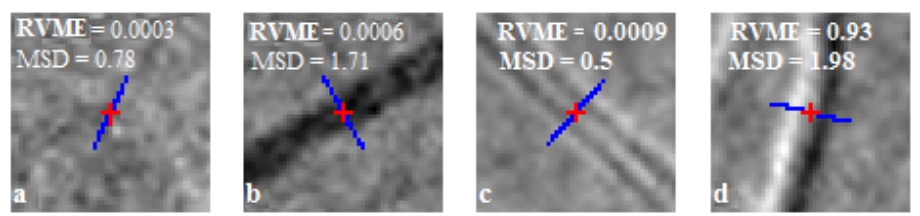

Fig. 4: Examples of difference image patches. Corresponding MSD (for the entire patch) and RVME values (at the red-cross locations) are shown on each patch.

to an MSD value as high as in case of a clear misalignment, while the RVME remains low. Figure 4c shows an example of a well-aligned vessel suffering from a clear difference in (de)focus, resulting in a significant MSD. In contrast, these examples show a very small value for the proposed RVME measure, indicating a very accurate registration. In case of actual registration errors, such as in figure 4d, the RVME is close to 1, corresponding to the expected value for a registration error of about the width of a vessel. To evaluate the robustness of the RVME measure to higher noise levels than the noise available in the normalized images which is $\sigma_{\text {noise }}^{2}=1$, a Gaussian noise was added to each of the registered images. Evaluation results at 5 randomly selected points show that the RVME measure barely changes with the noise in the registered image pairs (Fig.5).

\section{Discussion}

In this paper, a new way of quantitatively assessing the registration accuracy of images in which the vasculature provides the main landmarks has been proposed. An accuracy measure (RVME) which exploits the even and odd signal property of the $1 \mathrm{D}$ profile across the vessels in the difference image is defined and used to determine the registration accuracy relative to the width of a vessel. The RVME measure is translated to the spatial registration accuracy in pixels 

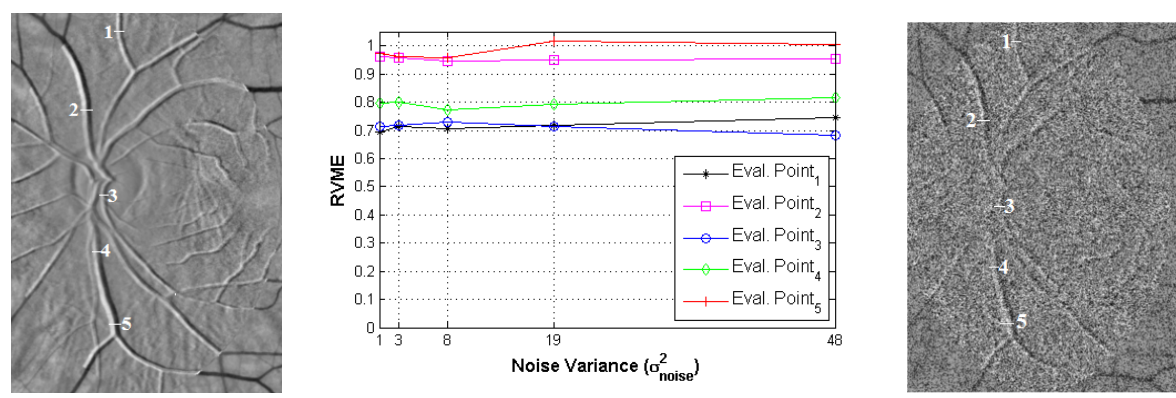

Fig. 5: Comparison of RVME measures at 5 randomly selected evaluation points on incorrectly registered image pair before (left image, $\sigma_{\text {noise }}^{2}=1$ ) and after adding Gaussian noise (right image, $\sigma_{\text {noise }}^{2}=48$ ).

by multiplication with the estimated vessel width, enabling an objective and quantitative registration accuracy assessment.

We demonstrated the method by applying it to registered red-free fundus images in order to quantify a misalignment error up to the full width of the widest vessel. Evaluation results showed that the RVME, in contrast with the MSD, does not depend on the intensity variation between registered image pairs, i.e. it is invariant to factors such as contrast, (de)focus, and noise (Fig. 4-5). It provides an excellent prediction of the imposed displacement (bias of -0.1 pixels and a standard deviation 0.9 pixels) in a controlled experiment.

\section{References}

1. Zitova, B., Flusser, J.: Image registration methods: a survey. Image and vision computing 21(11) (2003) 977-1000

2. Sotiras, A., Davatzikos, C., Paragios, N.: Deformable medical image registration: A survey. Medical Imaging, IEEE Transactions on 32(7) (2013) 1153-1190

3. Laliberte, F., Gagnon, L., Sheng, Y.: Registration and fusion of retinal images-an evaluation study. Medical Imaging, IEEE Transactions on 22(5) (2003) 661-673

4. Fitzpatrick, J.M.: Detecting failure, assessing success. In: Medical Image Registration. CRS Press (2001) 117-139

5. Christensen, G.E., Geng, X., Kuhl, J.G., Bruss, J., Grabowski, T.J., Pirwani, I.A., Vannier, M.W., Allen, J.S., Damasio, H.: Introduction to the non-rigid image registration evaluation project (nirep). In: WBIR. Springer (2006) 128-135

6. Adal, K.M., Ensing, R.M., Couvert, R., van Etten, P., Martinez, J.P., Vermeer, K.A., van Vliet, L.J.: A hierarchical coarse-to-fine approach for fundus image registration. In: Biomedical Image Registration. Springer (2014)

7. van Ginkel, M., Verbeek, P., van Vliet, L.J.: Improved orientation selectivity for orientation estimation. In: Proc. Scan. Conf. on Im. Anal. Volume 1. (1997) 533-537

8. Faas, F.G., van Vliet, L.J.: 3d-orientation space; filters and sampling. In: SCIA 2003. Lecture Notes in Computer Science. Vol. 2749 (2003) 36-42

9. Frangi, A., Niessen, W., Vincken, K., Viergever, M.: Multiscale vessel enhancement filtering. In: MICCAI. Lecture Notes in Computer Science. Vol. 1496 (1998) 130-137 